Journal of Computer Science 8 (6): 951-956, 2012

ISSN 1549-3636

(C) 2012 Science Publications

\title{
Efficient Star Topology based Multicast Key Management Algorithm
}

\author{
Saravanan, K. and T. Purusothaman \\ Department of CSE, Government College of Technology, Coimbatore, India
}

\begin{abstract}
Problem statement: Secure group communication is very important for many applications such as internet pay sites. It provides efficient delivery of identical data to only the customers in the group. In large and dynamic multicast groups, the group keys of members have to be changed frequently whenever the member leaves or joins. A common method is to apply a symmetric key that is used to encrypt the transmitted data. The rekeying cost scales linearly with the number of members in the group and cost of the rekeying process is the main issue. The tree-based architecture is commonly used to reduce the rekeying cost in terms of storage, transmission and computation. But it usually gives extra overhead to balance the tree which is in order to achieve logarithmic rekeying cost. Approach: The main aim was to use star topology based architecture to avoid the balancing and eliminate the rekeying processes and more over it was more secured by exchanging the secret key between only server and each group member. The features of proposed algorithm were that the private key was computed by individual member. Results: The burden of server was reduced and also there was no rekeying when a member leaves the group. The secret value of leaving member was not added in the encryption and so the private value could not be obtained after decryption. Conclusion: Proposed algorithm is simple and no rekeying when a member leaves and also reduces the computation and communication complexity.
\end{abstract}

Key words: Group communication, rekeying, secure multicast, key management

\section{INTRODUCTION}

In the modern technology world, network attacks have become more sophisticated and harder to identify the attack. When many applications like scalable chat services and streaming video, are expected to run over the Internet, the security is necessary in computing and communication became a necessity. The internet today provides less security for privacy and authentication of multicast packets. The number of applications using multicast increases day by day and so it need secure multicast services.

Multicast is an internetwork service which provides efficient delivery of data from a source to multiple receivers and also improve the bandwidth efficiency of the network. A common group key is necessary for individual members in the group for secure multicast communication. In general the group key management (Peyravian et al., 1999; Rafaeli and Hutchison, 2003; Zhu and Jajodia, 2003; Kim et al., 2005; Devi and Padmavathi, 2010; Sahar et al., 2010; Abdul-Rahman et al., 2011) can be divided into three categories (a) centralized key management (b) distributed key management (c) decentralized key management.

In all approaches (Harney and Muckenhirm, 1997; Waldvogel et al., 1999; Wong et al., 2000; Sherman and McGrew, 2003; Sahar et al., 2010) whenever a member joins or leaves the group or the members are static in nature, the group key has to be changed to achieve forward secrecy which assures that the newly joined members cannot decrypt the multicast data sent earlier before joining the group and assures that the former members cannot decrypt the communication after leaving the group.

In most of the key management protocol (Peyravian et al., 1999; Tu et al., 1999; Wong et al., 2000; Selcuk and Sidhu, 2002; Zhu and Jajodia, 2003; Kreishan, 2011; Mansouri et al., 2011) tree topology is used. Tree balancing is another issue when a member joins or leaves. The main drawback of tree topology is that number of overhead and cost for rekeying proportionately increase if the number of member increases. A huge database is necessary for storage and complexity also increases. Scalability is an issue in connection with the dynamic multicast members.

\section{MATERIALS AND METHODS}

The drawback of tree based architecture was overcome in SBMK (Lin et al., 2010) which uses starbased architecture in which the server computes a secret key and unicast to every user separately. But the drawbacks of these kinds of protocols are as follows:

Corresponding Author: Saravanan, K., Department of CSE, Government College of Technology, Coimbatore, India 
- It increases the load on the server

- Computational and communication complexities are increased

- If private key is computed and sent by a server to all the members then the private component of members may not be used for authentication

Our star topology based proposed algorithm has overcome the above problem:

- The total load on the server reduces because the private key is computed and sent by each user to server. So it reduces the load on the server

- The private component of members may be used for authentication

- It also reduces the computation complexity of server

- It gives a better rekeying performance than that of the key tree and there is no need to balance the tree

- Moreover our proposed scheme takes care of the important security requirements for secure group communication such as group secrecy, forward secrecy and backward secrecy

In this study, we propose an efficient star based key management algorithm for internet pay sites, which is relatively simple to implement.

The rest of the study describes the proposed scheme, derives the result with suitable illustration of proposed algorithm, discuss and compare the proposed algorithm with the existing algorithms and finally concludes the study and future work.

Proposed scheme: In the proposed star topology based algorithm, the individual member joining the group is allowed to choose prime numbers and compute their private key and the secret value of $\mathrm{N}$ computed is sent to the server by a secure unicast message. Thus the burden of server is reduced and also rekeying is totally reduced and also scalable for a large multicast group.

Key assignment phase: The steps of key assignment are as listed below:

Step 1: First the server authenticates the user who want to join the multicast group and also announce public value as e. It is common for the server as well as users.

Step 2: The individual user Mi randomly select two prime numbers $\mathrm{m}$ and $\mathrm{n}$ and calculate the product $\mathrm{Xi}=\operatorname{mi} \mathrm{x}$ ni and $\phi(\mathrm{Xi})=(\mathrm{mi}-1) \mathrm{x}(\mathrm{ni}-1)$.
Step 3: The private of key of individual member will be calculated (Rivest et al., 1978; Menezes et al., 1997; Sharma et al., 2011) by the user using the extended Euclidean algorithm to calculate a unique integer $d_{i}$ such that Eq. 1:

e $x d_{i} \equiv 1 \bmod \left(\phi\left(X_{i}\right)\right.$

where, $d_{i}>\phi\left(X_{i}\right)$

Step 4: The authenticated individual members send their $\mathrm{X}$ value to the server.

Step 5: The server verifies and accepts the $X_{i}$ value only if it is unique value from other members and hold the value of $X_{i}$ as secret.

\section{Message encryption:}

Step 1: When the server wants to send a secret message $\mathrm{P}$ to selected users in the multicast group $\mathrm{M}_{1}, \mathrm{M}_{2}, \mathrm{M}_{4}$, then the server uses e as well as the secrets of Members $\mathrm{X}_{1}, \mathrm{X}_{2}$ and $\mathrm{X}_{4}$

The encryption of the secret message is computed using the general formulae Eq. 2 (Lin et al., 2010):

$\mathrm{C}=(\mathrm{P})^{\mathrm{e}} \bmod \prod \mathrm{Xi}$

where, $\mathrm{Xi}$ includes the $\mathrm{X}$ value of members to whom the secret plain text has to be sent.

Step2: The server computes cipher text and sends a broadcast message to all the members of the group.

\section{Message decryption:}

Step 1: The individual member $\mathrm{Mi}$ receiving the cipher text $\mathrm{C}$ can use its private key di and his/her public parameter $\mathrm{Ni}$, to decrypt the plain text and obtain the secret and confidential message $\mathrm{P}$ using (Lin et al., 2010) the following formulae Eq. 3:

$\mathrm{Mi} \rightarrow \mathrm{P}=(\mathrm{C} \bmod \mathrm{Xi})^{\mathrm{di}} \bmod \mathrm{X}_{\mathrm{i}}$

Member joining: When a new member $\mathrm{Mn}+1$ want to join the group, the key server repeats the procedures similar to key assignment.

Step 1: First the server authenticates the user who want to join the multicast group and also announce public value as e .It is common for the server as well as users. 
Step 2: The individual user $\mathrm{M}_{\mathrm{i}+1}$ select two prime numbers $m$ and $n$ and calculate the product $\mathrm{X}_{\mathrm{i}+1}=\mathrm{m}_{\mathrm{i}+1} \times \mathrm{n}_{\mathrm{i}+1}$ and $\phi\left(\mathrm{X}_{\mathrm{i}+1}\right)=\left(\mathrm{m}_{\mathrm{i}+1}-1\right) \mathrm{x}$ $\left(n_{i+1}-1\right)$.

Step 3: The private of key (Rivest et al., 1978; Menezes et al., 1997; Sharma et al., 2011) of individual member will be calculated by the user using the extended Euclidean algorithm to calculate a unique integer $\mathrm{d} 0$ such that:

$$
\mathrm{e} \times \mathrm{d}_{\mathrm{i}+1}=1 \bmod \left(\phi\left(\mathrm{X}_{\mathrm{i}+1}\right)\right.
$$

Step 4: The newly joined member send their $X_{i+1}$ value to the server

Step 5: The server verify the $X_{i+1}$ and accept the $X_{i+1}$ only if it is unique value from other members and hold the value of $\mathrm{X}$ as secret

Members leaving: When a member Xi leaves the group, the key server just deletes the secret information $\mathrm{X}_{\mathrm{i}}$. Therefore, in the cipher text computation (Rivest et al., 1978; Menezes et al., 1997; Sharma et al., 2011) in Formula (2) removes the modulus operations with respect to $X_{i}\left(m_{i} \times n_{i}\right)$. Member $\mathrm{Mi}$, cannot decrypt the secrete message because $\mathrm{Xi}$ is not added in cipher text calculation. Hence both forward and backward secrecy is maintained. The pair of prime numbers of a leaving member cannot be reassigned to new user joining the group. So there is no need for rekeying even if the members of multicast group change.

\section{RESULTS}

Illustration of the proposed algorithm with suitable examples and the result obtained is discussed in this section.

Key assignment phase: The steps of key assignment are as listed below:

Step 1: First the server authenticate the user who want to join the multicast group and also announce public value as $\mathrm{e}=103$. It is common for the server as well as users.

Step 2: The individual user Mi selects two prime numbers mi and ni randomly and also compute their $\mathrm{Xi}=$ mi $\mathrm{x}$ ni and $\Phi\left(\mathrm{X}_{\mathrm{i}}\right)=\left(\mathrm{m}_{\mathrm{i}}-1\right) \times\left(\mathrm{n}_{\mathrm{i}}-1\right)$ :

$M_{1}$ selects $m_{1}=163$ and $n 1=227$

computes $X_{1}=37001$ and $\phi\left(X_{1}\right)=36612$
$\mathrm{M}_{2}$ selects $\mathrm{m}_{2}=181$ and $\mathrm{n} 2=233$

computes $X_{2}=42173$ and $\phi\left(X_{2}\right)=41760$

$M_{3}$ selects $m_{3}=163$ and $n 3=199$

computes $X_{3}=32437$ and $\phi\left(X_{3}\right)=32076$

$\mathrm{M}_{4}$ selects $\mathrm{m}_{4}=137$ and $\mathrm{n} 4=173$

computes $\mathrm{X}_{4}=23701$ and $\phi\left(\mathrm{X}_{4}\right)=23392$

$\mathrm{M}_{5}$ selects $\mathrm{m}_{5}=223$ and $\mathrm{n} 5=211$

computes $\mathrm{X}_{5}=47053$ and $\phi\left(\mathrm{X}_{5}\right)=46620$

$M_{6}$ selects $m 6=251$ and $n 6=191$

computes $\mathrm{X}_{1}=47941$ and $\phi\left(\mathrm{X}_{6}\right)=47500$

Step 3: The private of key of individual member is calculated by each user using the extended Euclidean algorithm used in RSA algorithm:

$103 \times \mathrm{d}_{1} \equiv 1 \bmod \left(\phi\left(\mathrm{X}_{1}\right) \equiv 1 \bmod 36612\right.$ and $\mathrm{d} 1=16351$

$103 \times \mathrm{d}_{2} \equiv 1 \bmod \left(\phi\left(\mathrm{X}_{2}\right) \equiv 1 \bmod 41760\right.$ and $\mathrm{d} 2=6487$

$103 \times \mathrm{d}_{3} \equiv 1 \bmod \left(\phi\left(\mathrm{X}_{3}\right) \equiv 1 \bmod 32076\right.$ and $\mathrm{d} 3=28339$

$103 \times \mathrm{d}_{4} \equiv 1 \bmod \left(\phi\left(\mathrm{X}_{4}\right) \equiv 1 \bmod 23392\right.$ and $\mathrm{d} 4=6359$

$103 \times \mathrm{d}_{5} \equiv 1 \bmod \left(\phi\left(\mathrm{X}_{5}\right) \equiv 1 \bmod 46620\right.$ and $\mathrm{d} 5=16747$

$103 \times \mathrm{d}_{6} \equiv 1 \bmod \left(\phi\left(X_{6}\right) \equiv 1 \bmod 47500\right.$ and $\mathrm{d} 6=2767$

Step 4: The individual members communicate their $\mathrm{X}$ value to the server:

$X 1=37001, \quad X 2=42173, \quad X 3=32437 \quad X 4=23701$

$\mathrm{X} 5=47053, \quad \mathrm{X} 6=47941$

Step 5: Server verifies the secret value $\mathrm{Xi}$ of individual users and accepts the $\mathrm{X}$ only if it is unique value from other members and hold the value of $\mathrm{Xi}$ as secret.

\section{Multicast communication:}

Step 1: Assume there are 6 members X1, X2, X3, X4, $\mathrm{X} 5, \mathrm{X} 6$ in the multicast group. When the server wants to send a secret message $P=5$ to all members in the multicast group, then the server uses its public value $\mathrm{e} 0$ as well as the secrets of Members X1, X2, X3, X4, X5,X6 in the encryption formulae

The encryption of the secret message is computed using the general formulae:

$$
\begin{gathered}
C=5^{103} \bmod \left(X_{1} \times X_{2} \times X_{3} \times X_{4} \times X_{5} \times X_{6}\right) \\
=5^{103} \bmod (37001 \times 42173 \times 32437 \times 23701 \\
\times 47053 \times 47941) \\
=1749630034980094709227313040
\end{gathered}
$$

Step2: The server computes cipher text $\mathrm{C}$ and sends a broadcast message to all the members of the group 
Step3: All six members in the multicast group can decrypt the secret message $\mathrm{P}$ from the cipher text received. The secret message is decoded as follows:

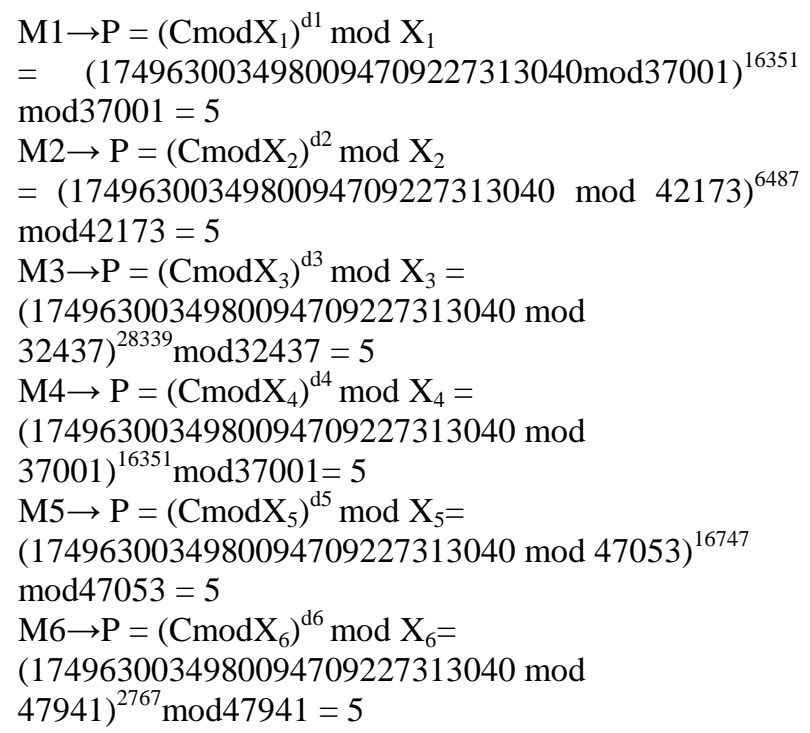

Members joining: When two members M7 and M8 want to join the multicast group, the key server repeats the formalities similar to key assignment.

Step 1: First the server authenticates the users M7 and M8 who want to join the multicast group and also inform the public value as $\mathrm{e}=103$.It is common for the server as well as users.

Step 2: Member M7 selects two prime numbers $(\mathrm{m} 7=149, \mathrm{n} 7=191)$ and M8 selects two prime numbers $(\mathrm{m} 8=199, \mathrm{n} 8=179)$ randomly and also compute their.

$\mathrm{X}_{\mathrm{i}}=\mathrm{m}_{\mathrm{i}} \times \mathrm{n}_{\mathrm{i}}$ and $\phi\left(\mathrm{X}_{\mathrm{i}}\right)=\left(\mathrm{m}_{\mathrm{i}}-1\right) \times\left(\mathrm{n}_{\mathrm{i}}-1\right)$

$\mathrm{M}_{7}$ selects $\mathrm{m}_{1}=149$ and $\mathrm{n} 1=191$ computes $\mathrm{X}_{7}=28459$ and $\phi\left(\mathrm{X}_{7}\right)=28120$

$M_{8}$ selects $m_{2}=199$ and $n 2=179$ computes $X_{8}=35621$ and $\phi\left(\mathrm{X}_{8}\right)=35244$

Step 3: The private of key of individual member M7 and M8 is calculated by each user using the extended Euclidean algorithm used in RSA algorithm:

$103 \times \mathrm{d} 7 \equiv 1 \bmod (\phi(X 7) \equiv 1 \bmod 28120$ and $\mathrm{d} 7=27847$

$103 \times \mathrm{d} 8 \equiv 1 \bmod (\phi(X 8) \equiv 1 \bmod 35244$ and $\mathrm{d} 8=13687$

Step 4: Member M7 and M8 inform their $\mathrm{X}$ values to the server
Step 5: Now the server will add M7 and M8 in the database and also when it is sending a new secret value, it will add M7 and M8 in the cipher text formulae

Step 6: Suppose if the server wants to send a new secret message $P=342$ to all the members $X_{1}, X_{2}, X_{3}$, $\mathrm{X}_{4}, \mathrm{X}_{5}, \mathrm{X}_{6}, \mathrm{X}_{7}$ and $\mathrm{X}_{8}$, it will compute new cipher text using the formulae in Eq. 2:

$\mathrm{C}=342^{103} \bmod \left(\mathrm{X}_{1} \times \mathrm{X}_{2} \times \mathrm{X}_{3}\right.$

$\left.\times \mathrm{X}_{4} \times \mathrm{X}_{5} \times \mathrm{X}_{6} \times \mathrm{X}_{7} \times \mathrm{X}_{8}\right)$

$=342^{103} \bmod (37001 \times 42173 \times 32437 \times 23701 \times$

$47053 \times 47941 \times 28459 \times 35621)$

$=3539761046220992139642094726449599963$

Step7: The existing group members $X_{1}, X_{2}, X_{3}, X_{4}, X_{5}$, $\mathrm{X}_{6}$ use their exiting private key to decrypt the cipher text to get the new secret message and the newly added two members $\mathrm{X}_{7}$ and $\mathrm{X}_{8}$ use their private key to decrypt the secret message as given below:

$\mathrm{M} 1 \rightarrow \mathrm{P}=(\mathrm{CmodX} 1)^{\mathrm{d} 1} \bmod \mathrm{X}_{1}$

$=(3539761046220992139642094726449599963 \mathrm{mod}$ $37001)^{16351} \bmod 37001=342$

$\mathrm{M} 2 \rightarrow \mathrm{P}=(\mathrm{CmodX} 2)^{\mathrm{d} 2} \bmod \mathrm{X}_{2}$

$=(3539761046220992139642094726449599963 \mathrm{mod}$ $42173)^{6487} \bmod 42173=342$

$\mathrm{M} 3 \rightarrow \mathrm{P}=(\mathrm{CmodX} 3)^{\mathrm{d} 3} \bmod \mathrm{X}_{3}$

$=(3539761046220992139642094726449599963 \mathrm{mod}$ $32437)^{28339} \bmod 32437=342$

$\mathrm{M} 4 \rightarrow \mathrm{P}=(\mathrm{CmodX} 4)^{\mathrm{d} 4} \bmod \mathrm{X}_{4}$ $=(3539761046220992139642094726449599963 \mathrm{mod}$ 23701) $)^{16351} \bmod 23701=342$

$\mathrm{M} 5 \rightarrow \mathrm{P}=(\mathrm{CmodX} 5)^{\mathrm{d} 5} \bmod \mathrm{X}_{5}$ $=(3539761046220992139642094726449599963 \mathrm{mod}$ $47053)^{16747} \bmod 47053=342$

$\mathrm{M} 6 \rightarrow \mathrm{P}=(\mathrm{C} \bmod \mathrm{X} 6)^{\mathrm{d} 6} \operatorname{modX} \mathrm{X}_{6}$

$=(3539761046220992139642094726449599963 \mathrm{mod}$ $47941)^{2767} \bmod 47941=342$

$\mathrm{M} 7 \rightarrow \mathrm{P}=(\mathrm{CmodX} 7)^{\mathrm{d} 7} \bmod \mathrm{X}_{7}$ $=(3539761046220992139642094726449599963 \mathrm{mod}$ $28459)^{27847} \bmod 28459=342$

$\mathrm{M} 8 \rightarrow \mathrm{P}=(\mathrm{CmodX} 8)^{\mathrm{d} 8} \bmod \mathrm{X}_{8}$

$=(3539761046220992139642094726449599963 \mathrm{mod}$ $35621)^{13687} \bmod 35621=342$

Members leaving: When two members $M_{5}$ and $M_{6}$ leaves the group, the key server just deletes the secret information of $X_{5}$ and $X_{6}$ correspond to $M_{5}$ and $M_{6}$.

Step1: In the cipher text computation in Formula (2) removes the modulus operations with respect to 
$X_{5}=47053$ and $X_{5}=47941$ if the server wants to send a new secret message $P=25$ to members $X_{1}$, $\mathrm{X}_{2}, \mathrm{X}_{3}, \mathrm{X}_{4}, \mathrm{X}_{7}$ and $\mathrm{X}_{8}$ :

$\mathrm{C}=342^{103} \bmod \left(\mathrm{X}_{1} \times \mathrm{X}_{2} \times \mathrm{X}_{3} \times \mathrm{X}_{4} \times \mathrm{X}_{7} \times \mathrm{X}_{8}\right)$ $=5508369876121780915818034295$

Step 2: Using the private keys, the members M1, M2, M3, M4, M7, M8 can decrypt the secret message $\mathrm{P}=25$

Members M5 and M6 will get different secret message as 25057 and 11127 respectively, which is different from the actual one.

The members $M_{5}$ and $M_{6}$ cannot decrypt the secret message because $X_{5}$ and $X_{6}$ is not added in the cipher text computation. Hence both forward and backward secrecy is maintained. When a member leaves the group, the server would not allow a new member to select the same pair of prime number. So there is no need for rekeying even if the members of multicast group change.

\section{DISCUSSION}

Complexity analysis: The secret $\mathrm{Xi}$ generated by the individual authenticated user is hold by server as a confidential one. It is known only to the corresponding users and server.

Difficulty for unauthorised member try to deduce the private key and secret value: The security of our proposed algorithm depends on the secret value and private key of individual users. It is not possible for the unauthorized person to derive the private key di from the public parameter e. It is extremely difficult for the adversary to derive the private key from the public parameter e alone. Moreover the secret value $\mathrm{X}$ is unique and the number of digit may also vary for every user.

Preventing the unauthorized access: If a member is not authenticated by the server, the server will not add the secret value of $X$ in the cipher text calculation and unauthorized member will get different value when he tries to decrypt the encrypted message. So it is more secured.

Performance analysis based on complexity comparison of various key management schemes: Table 1-4 provides the comparative analysis of the various protocols. It shows that every protocol achieves unique results when applying different techniques. Some protocols achieve exceptionally better results than others do. By comparing the table, we can clearly understand that the bottleneck of server is avoided by reducing total no of keys managed by server in our proposed algorithm. It is also smaller when compared with LKH, OFT and SBMK algorithm (Kim et al., 2005; Lin et al., 2010; Abdul-Rahman et al., 2011).

Only one multicast message will be send to the group when a member joins and no message is send when a member leaves the group. So there is no need for rekeying when a member leaves also the rekeying overhead is less compared with LKH and OFT (Kim et al., 2005; Abdul-Rahman et al., 2011).

The proposed algorithm achieves better results for storage, communication, computation and processing on both server and user. The computation cost of server is greatly reduced by allowing the users to calculate their private key and secret values compared with other techniques.

The cost of encryption when a member joins the group is 1 and the cost of encryption when a member leaves the group.

From the tables we can easily understand that proposed protocol is more suitable for a dynamic users and storage cost of server is reduced (Lin et al., 2010) and distributed to the users.

Table 1: Communication cost

\begin{tabular}{lll}
\hline Protocols & $\begin{array}{l}\text { Join } \\
\text { multicast }\end{array}$ & $\begin{array}{l}\text { Leave } \\
\text { unicast }\end{array}$ \\
\hline LKN 2 LOG -1 & Log $n$ & Log $n+1$ \\
One way function tree & $\log n+1$ & 0 \\
SBMK & 1 & 0 \\
Proposed protocol & 1 & \\
\hline
\end{tabular}

Table 2: Computation cost

\begin{tabular}{lll}
\hline Protocols & Join & Leave \\
\hline LKH & $2 \log \mathrm{n}-1$ & $2 \log \mathrm{n}$ \\
One way function tree & $\log \mathrm{n}+1$ & $\log \mathrm{n}+1$ \\
SBMK & 1 & 0 \\
Proposed protocol & 1 & 0 \\
\hline
\end{tabular}

Table 3: No of rekey messages

\begin{tabular}{lll}
\hline & No of rekey messages needed \\
Protocols & Join & Leave \\
\hline LKH & $\mathrm{d}+1$ & $2 \mathrm{~d}$ \\
One way function tree & $\mathrm{d}+1$ & $\mathrm{~d}+1$ \\
SBMK & 1 & 0 \\
Proposed Protocol & 1 & 0 \\
\hline
\end{tabular}

Table 4: Key storage during join and leave operations

\begin{tabular}{lll}
\hline Protocols & Server & User \\
\hline LKH & $2 n$ & $\log n+1$ \\
One way function tree & $2 n$ & $2 \log n+$ \\
SBMK & $2 n+2$ & 3 \\
Proposed protocol & $n+2$ & 3 \\
\hline
\end{tabular}




\section{CONCLUSION}

In this study, an efficient Star Topology based Multicast Key Management algorithm is proposed and implemented which produces better results than the existing protocols in terms of less computational, communication and storage costs. The proposed star based architecture reduces the rekeying overhead. The private key of the users are computed by the individual and so it can be used for authentication also.

The computation complexity of the server is totally reduced in the new protocol. It is also scalable and easy to implement when the number of users are very high and dynamic in nature. As future scope of work, it may be extended for bulk member join and leaves.

\section{REFERENCES}

Abdul-Rahman, H., A.M. Alashwal and Z.H. Jamaludin, 2011. Implementation and methods of project learning in quantity surveying firms: Barriers, enablers and success factors. Am. J. Econ. Bus. Admin., 3: 430-438. DOI: 10.3844/ajebasp.2011.430.438

Devi D.S. and G. Padmavathi, 2010. Secure multicast key distribution for mobile adhoc networks. Int. J. Comput. Sci. Inform. Security, 7: 218-222.

Harney, H. and C. Muckenhirm, 1997. Group Key Management Protocol (GKMP) architecture. SPARTA, Inc.

Kim, H., S.M. Hong, H. Yoon and J.W. Cho, 2005. Secure group communication with multiplicative one-way functions. Proceedings of the IEEE International Conference on Information Technology: Coding and Computing, Apr. 4-6, IEEE Xplore Press, Washington, DC, USA., pp: 685-690. DOI: 10.1109/ITCC.2005.252

Kreishan, F.M., 2011. Economic growth and unemployment: An empirical analysis. J. Soc. Sci., 7: 228-231. DOI: 10.3844/jssp.2011.228.231

Lin, I.C., S.S. Tang and C.M. Wang, 2010. Multicast key management without rekeying processes. Comput. J., 53: 940-950. DOI: 10.1093/comjnl/bxp060

Mansouri, M., A. Ganguly and A. Mostashari, 2011. Evaluating agility in extended enterprise systems: A transportation network case. Am. J. Eng. Applied Sci., 4: 142-152. DOI: 10.3844/ajeassp.2011.142.152

Menezes, A.J., P.C.V. Oorschot and S.A. Vanstone, 1997. Handbook of Applied Cryptography. 1st Edn., CRC Press, Boca Raton, Fla., ISBN: 0849385237, pp: 780.
Peyravian, M., S.M. Matyas and N. Zunic, 1999. Decentralized group key management for secure multicast communications. Comput. Commun., 22: 1183-1187. DOI: 10.1016/S0140-3664(99)00121-8

Rafaeli, S. and D. Hutchison, 2003. A survey of key management for secure group communication. J. ACM Comput. Surveys, 35: 309-329. DOI: 10.1145/937503.937506

Rivest, R.L., A. Shamir and L. Adleman, 1978. A method for obtaining digital signatures and publickey cryptosystems. Mag. Commun.. ACM., 21: 120-126. DOI: $10.1145 / 359340.359342$

Sahar, N.B.M., S. Ardi, S. Kazuhiko, M. Yoshiomi and M. Hirotsugu, 2010. HAZOP analysis management system with dynamic visual model aid. Am. J. Applied Sci., 7: 943-948. DOI: 10.3844/ajassp.2010.943.948

Selcuk, A.A. and D. Sidhu, 2002. Probabilistic optimization techniques for multicast key management. Comput. Netw., 40: 219-234. DOI: 10.1016/S1389-1286(02)00252-9

Sharma, S., P. Sharma and R.S. Dhakar, 2011. RSA algorithm using modified subset sum cryptosystem. Proceedings of the 2nd International Conference on Computer and Communication Technology (ICCCT), Sep. 15-17, IEEE Xplore Press, Allahabad, pp: 457-461. DOI: 10.1109/ICCCT.2011.6075138

Sherman, A.T. and D.A. McGrew, 2003. Key establishment in large dynamic groups using oneway function trees. IEEE Trans. Software Eng., 29: 444-458. DOI: 10.1109/TSE.2003.1199073

Tu, F.K., C.S. Laih and H.H. Tung, 1999. On key distribution management for conditional access system on pay-TV system. IEEE Trans. Consumer Elect., 45: 151-158. DOI: 10.1109/30.754430

Waldvogel, M., G. Caronni, D. Sun, N. Weiler and B. Plattner, 1999. The versakey framework: Versatile group key management. IEEE J. Selected Areas Commun., 17: 1614-1631. DOI: 10.1109/49.790485

Wong, C.K., M. Gouda and S.S. Lam, 2000. Secure group communications using key graphs. IEEE/ACM Trans. Netwo., 8: 16-30. DOI: 10.1109/90.836475

Zhu, S. and S. Jajodia, 2003. Scalable Group rekeying for secure multicast: A survey. Lecture Notes Comput. Sci., 2918: 833-833. DOI: 10.1007/978-3540-24604-6_1 